Ein neues Kern-Isomer von $\mathbf{T}=\mathbf{3 , 9} \mathrm{min}$ beim Selen

Von A. Flammersfeld und Chr. Ythier Max-Planck-Institut für Chemie, Mainz

(Z. Naturforschg. 5 a, 401 [1950]; eingeg. am 26. Juni 1950)

Selen, das mit Neutronen bestrahlt wird, zeigt ein kompliziertes radioaktives Verhalten wegen der Vielzahl der auftretenden Halbwertszeiten. Außer den länger bekannten Aktivitäten von ${ }^{75} \mathrm{Se}(127 \mathrm{~d}),{ }^{11} \mathrm{Se}(18 \mathrm{~min}),{ }^{81} \mathrm{Se}^{*}(57 \mathrm{~min})$ und ${ }^{83 \mathrm{Se}}(30 \mathrm{~min})$ wurden neuerdings von Arnold und $\mathrm{S} \mathrm{u}$ g a rm a ${ }^{1}{ }^{1}$ zwei weitere, durch langsame Neutronen erhältliche Aktivitäten ${ }^{(83)} \mathrm{Se}(1,1 \mathrm{~min})$ und ${ }^{77} \mathrm{Se}^{*}(17,5 \mathrm{sec})$ aufgefunden.

In der vorliegenden Arbeit sollte dieses Isomer näher untersucht werden, wozu elementares Selen in dünner Schicht $\left(5 \mathrm{mg} / \mathrm{cm}^{2}\right)$ mit $\mathrm{Be}+\mathrm{D}$ - und $\mathrm{Li}+\mathrm{D}$-Neutronen unserer Hochspannungsanlage $\left(E_{\mathrm{D}}=1,4 \mathrm{MeV}\right)$ bestrahlt und in einem Schleusenzählrohr mit besonders dünnem Fenster $(0,2 \mu$ Zaponlack) gemessen wurde.

Im Laufe der Untersuchung stellte sich heraus, daß eine weitere bisher unbekannte Aktivität durch Neutronen beim Selen entsteht, die eine Halbwertszeit von $T=3,9 \pm 0,1 \mathrm{~min}$ besitzt und eine sehr weiche Strahlung aussendet.

Die Erzeugung des 3,9-min-Körpers ist sowohl durch unverlangsamte wie durch verlangsamte $\mathrm{Be}+\mathrm{D}$-Neutronen möglich, wobei im letzteren Falle eine erhebliche Verstärkung stattfindet. Das bedeutet also, daß er hierbei im wesentlichen durch $(\mathrm{n}, \gamma)$-Prozeß entsteht. Die Tatsache, $\mathrm{da} ß$ er auch durch unverlangsamte $\mathrm{Be}+\mathrm{D}$-Neutronen gebildet wird, bedeutet nicht, daß auch ein $(n, n)-$ Proze $\beta$ stattfinden muß, da auch die 57-min- und 18-min-Periode mit unverlangsamten $\mathrm{Be}+\mathrm{D}-\mathrm{Neutronen}$ sehr stark werden, obwohl sie sicher nur durch $(\mathrm{n}, \gamma)$-Prozeß entstehen. Die Intensität des 3,9-min-Körpers ist derart, da $\beta$ er bei kurzer Bestrahlung (z. B. 3 min) mit unverlangsamten $\mathrm{Be}+\mathrm{D}-$ Neutronen nach Abklingung der sehr starken 17,5-sec-Aktivität etwa 3 -mal so intensiv ist wie die 18-min- und 57-min-Körper. Noch deutlicher tritt die neue Aktivität bei Bestrahlung des Selens mit unverlangsamten $\mathrm{Li}+\mathrm{D}-\mathrm{Neutronen}$ auf. Ebenso wie die 57-minAktivität wird sie hier offenbar durch einen (n, 2n)-Prozeß gebildet, während der 18-min-Körper im Einklang mit den Angaben in der Literatur nicht merkbar wird.

Die Strahlung des 3,9-min-Körpers ist in Aluminium sehr stark absorbierbar, sie besteht aus Elektronen; $\gamma$-Strahlen sind keine vorhanden. Die Reichweite der Elektronen ist nur mäßig genau bestimmbar, weil nach Absorption der 3,9-min-Komponente noch zu viel andere Aktivitäten vorhanden sind. Sie beträgt etwa $9,5 \mathrm{mg} / \mathrm{cm}^{2} \mathrm{Al}$ entsprechend einer Energie von 0,09 $\pm 0,01 \mathrm{MeV} 2$.

Es wurden drei reine Selenproben verschiedener Herkunft untersucht, die alle die 3,9-min-Aktivität in derselben Intensität ergaben. Hieraus und der mit den übrigen Se-Aktivitäten vergleichbaren Intensität muß geschlossen werden, daß es sich nicht um eine Verunreinigung han-

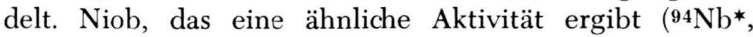

1 J. R. Arnold u. N. S u garman, J. chem. Physics 15, 703 [1947].

2 A. F la m mersfeld, Z. Naturforschg. 2a, 370 [1947].
$T=6,6 \mathrm{~min}, E_{\beta}=0,041 \mathrm{MeV}$ ) wurde durch gleichzeitige Bestrahlung von Se- und Nb-Präparaten ausgeschlossen; es zeigte sich, daß der Wirkungsquerschnitt von $\mathrm{Nb}$ mindestens um den Faktor 100 zu klein ist, um als Verunreinigung im Selen die neue Aktivität vortäuschen zu können. Andere Elemente, die ähnliche Halbwertszeiten mit derart geringer Energie ergeben, sind nicht bekannt, so daß auch aus diesem Grund keine Möglichkeit besteht, zur Erklärung eine Verunreinigung anzunehmen.

Bei der 3,9-min-Aktivität kann es sich offenbar nur um einen isomeren Zustand eines Selen-Isotops handeln, der durch Isomeren-Übergang in den Grundzustand übergeht. Die Anregungsenergie von $0,090 \mathrm{MeV}$ ist mit der Halbwertszeit dann durchaus verträglich. Die Zuordnung $\mathrm{zu}$ einer bestimmten Massenzahl ist noch nicht möglich. Die Tatsache, daß sowohl $(\mathrm{n}, \gamma)$ - wie $(\mathrm{n}, 2 \mathrm{n})$-Prozesse die 3,9-min-Aktivität ergeben, macht eine Zuordnung zu ${ }^{81} \mathrm{Se}^{*},{ }^{77} \mathrm{Se}^{*}$ oder zu ${ }^{79} \mathrm{Se}^{*}$ möglich.

In den ersteren Fällen handelte es sich also jeweils um ein zweites Isomer zu ${ }^{81} \mathrm{Se}$ bzw. ${ }^{77} \mathrm{Se}$, im letzteren Fall aber um ein Isomer zum noch unbekannten ${ }^{79} \mathrm{Se}$, das vermutlich sehr langlebig ist.

Bemerkenswert ist noch, daß die Auffindung der 3,9min-Aktivität vielleicht wichtig für einen Teil der Diskrepanzen bei den Angaben für die Halbwertszeit des 18-min-Körpers ist; möglicherweise ist der neueste Wert ${ }^{3}$ von $T=13,6 \mathrm{~min}$ durch eine geringe Beimischung zustande gekommen. Bei genügender Vorschaltung von Absorptionsfolien wurde hier immer $T=18 \pm 1 \mathrm{~min}$ gemessen.

Ferner wurde noch die 17,5 -sec-Aktivität des $77 \mathrm{Se}^{*}$ untersucht, die bei allen Bestrahlungsarten sehr intensiv wird. Die Elektronen, die bei dem isomeren Übergang ausgesandt werden, haben eine Reichweite von $30 \mathrm{mg} / \mathrm{cm}^{2}$ $\mathrm{Al}$ entspr. einer Energie von $0,165 \mathrm{MeV}$. Dieser Wert stimmt befriedigend überein mit einer Bestimmung von Gide on und Mitarbb. ${ }^{4}$, die $0,135 \mathrm{MeV}$ fanden, und der Energie der nicht umgewandelten $\gamma$-Strahlung von $\approx 0,150 \mathrm{MeV} 1$. Die Halbwertszeit wurde neu bestimmt zu $17,4 \pm 0,15$ sec.

Die von A rnold und S u ga r m a n gefundene Halbwertszeit von $T=1,1 \mathrm{~min}$ des ${ }^{(83)} \mathrm{Se}$ (große $\beta$-Energie, Nachweis hinter Al-Absorber zur Schwächung der weniger energiereichen anderen Aktivitäten) konnte ebenfails bestätigt werden.

${ }^{3} \mathrm{H}$. Wäffler u. O. Hirzel, Helv. physica Acta 21, 200 [1948].

4 D. M. Gideo n, W. C. Miller u. B. Waldm a n, Physic. Rev. 75, 329 (abs. A 8) [1949].

\section{Über die Zuordnung des 1,1-Stunden-Bleis}

Von $\mathrm{Kl}$ aus Geiger

Max-Planck-Institut für Chemie, Mainz

(Z. Naturforschg. 5 a, 401-403 [1950]; eingeg. am 12. Juni 1950)

Bei Bestrahlung von Blei mit Neutronen fanden de $\mathrm{Vr}$ ries und D i e m e ${ }^{1}$ eine Bleiaktivität von $1,3 \mathrm{~h}$ Halbwertszeit, die später von $\mathrm{Fajans}$ und Voigt 2 ge-

1 H. de Vries u. G. Diemer, Physica 6, 599 [1939].

2 K. Fajans u. A. F. Voigt, Physic. Rev. 60, 619 [1941]. 